Dieses Dokument ist eine Zweitveröffentlichung (Verlagsversion) / This is a self-archiving document (published version):

\author{
Alexander Lasch \\ Überlegungen zur 'Logikı der Sammlung und zur Relationierung von \\ Einzeltexten in Jakob Freys Gartengesellschaft (1557) \\ Erstveröffentlichung in / First published in: \\ Beate Kellner, et. al., Hrsg. Erzählen und Episteme. Berlin/New York: De Gruyter 2011, S. \\ 267 - 285. ISBN 978-3-484-97088-5. \\ DOI: https://doi.org/10.1515/9783484970885.267
}

Diese Version ist verfügbar / This version is available on:

https://nbn-resolving.org/urn:nbn:de:bsz:14-qucosa2-748393 


\title{
Überlegungen zur `Logikı der Sammlung und zur Relationierung von Einzeltexten in Jakob Freys Gartengesellschaft (1557)
}

\author{
1.
}

Die Gartengesellschaft wird in der germanistischen Forschung traditionell als Schwanksammlung oder Schwankbuch bezeichnet: ${ }^{1}$ Sie wird damit ebenso wie das Rollwagenbüchlein Jörg Wickrams, der Wegkürzer des Martin Montanus oder Michael Lindeners Katzipori als ein Buch verstanden, in dem vorwiegend schwankhafte Einzeltexte zusammengestellt sind - und zwar ohne dass der Kompilator sie einem der verfuigbaren Ordnungsmodelle unterwerfen würde. $\mathrm{Ob}$ es sich dabei lediglich um eine medial-materielle Einheit des `Erzählvorrats` handelt, oder ob und inwiefern dieser Zusammenstellung eine >Logikı der Sammlung mit bestimmten semantischen Konsequenzen innewohnt, ist oft diskutiert worden. Mein Beitrag möchte dieser Frage, angeregt von Überlegungen zur »Hermeneutik des Sammlers «, ${ }^{2}$ noch einmal am Beispiel der Gartengesellschaft nachgehen.

Der Buchdruck vervielfältigt nicht unmittelbar den Wissensbestand, sondern dessen Trägermaterial; die Einführung des Drucks mit beweglichen Lettern ist in erster Linie eine Medienrevolution. Der mediale Umbruch setzt nicht sogleich ıneues` Wissen frei, sondern zunächst wird das vorhandene Wissen in neuen Formen präsentiert und technisch verfügbar gemacht. Er begünstigt jedoch eine Verschiebung von rhetorischen zu topologischen Ordnungsmustern des Wissens. ${ }^{3}$ Tendenziell werden dabei »lebensweltliche" ebenso wie "gelehrte» Speicher, die durch die "mehr oder minder elaborierten Register der Rhetorik strukturiert« sind, ${ }^{4}$ unter funktionalen Gesichtspunkten umgebaut. Mit dem Buchdruck, der tradiertes Wissen

\footnotetext{
1 Dieckow 1996, S. 79f. Beide Termini verschweigen, dass nicht allein `Schwänke` in den Sammlungen aufgenommen sind, sondern auch andere kurzepische und sogar nicht-narrative Texttypen. Um diesem Charakeristikum der Sammlungen Rechnung zu tragen und das "Begriffschaos» (Straßner 1978, S. 3) um den Begriff >Schwank zu vermeiden, bietet sich der Terminus Prosaerzählsammlung an (vgl. Dieckow 1996, S. 85, und Ziegeler 2003, S. 409).

${ }^{2}$ Cahn 1991

3 Vgl. Friedrich 2004.

${ }^{4}$ Und zwar durch »Topiken mit ihren Orten« und »Memorialtechniken mit ihren Erinnerungsräumen« (ebd., S. 10). Vgl. zu ersteren Schmidt-Biggemann 1983, zu letzteren Carruthers 1990.
} 
technisch reproduzierbar macht und breit zur Verfügung stellt, wird die Durchsetzung funktionaler Ordnungsmodelle und Erschließungsmodalitäten befördert. Imperativ des Ordnens und Erschließens ist damit $>$ Finde! $<$ und nicht $>$ Wisse! $\measuredangle .^{5}$ Besonders deutlich zeigt sich dieser Wandel im Übergang vom Codex zum gedruckten Buch gerade auch an den Veränderungen der Ordnungsmodelle und Erschließungsmodalitäten von Sammlungen und Kompilationen.

Durch die Möglichkeiten der neuen Träger freilich werden sekundär auch qualitative Veränderungen des Wissens verstärkt und verursacht. Zwar wird das tradierte, nach rhetorischen Mustern geordnete Wissen nicht erst durch Humanisten und Reformatoren umgeschrieben, aber erst dann treten auch immer deutlicher Geltungskonkurrenzen wunterschiedlicher Relevanzkriterien in Bezug auf die Ordnung des Wissens « hervor. ${ }^{6}$ Der Buchdruck ermöglicht es, solche heterogenen Wissensbestände in rascher Folge und großer Menge zu verbreiten. Ein Indiz hierfür kann die Erfolgsgeschichte der kleineren Erzählformen in der Frühen Neuzeit sein, die nun nicht mehr selbstverständlich als Teil und Repräsentation einer universalen Ordnung gelten, sondern zunehmend die Singularität und Kontingenz des Erzählten ausstellen. Dabei kann ein andersartiges Wissen präsentiert werden, das zumindest potenziell geeignet ist, traditionelle universale Geltungsansprüche zu unterminieren. Das in der Frühen Neuzeit zu beobachtende Nebeneinander von verschiedenen Präsentationsformen unterschiedlich zu bewertender Wissensbestände führt, befördert durch die Interessen des sich etablierenden Buchmarktes, zum Relevanzverlust tradierter Ordnungsmodelle, während gleichzeitig alternative Formen der Wissenspräsentation aufgewertet werden.

Ein Relevanzverlust tradierten Wissens und tradierter Wissensordnungsmodelle kann sich im Zuge dieser Transformationen auch als eine Konsequenz der Praxis des Zusammentragens selbst einstellen, denn es liegt in der >Logik der Sammlung, dass ihr stets noch Fehlendes hinzugefuigt werden könnte. ${ }^{7}$ Die Expansion der Sammlung führt aber nicht unbedingt zu einem Wissensgewinn; sie kann auch lediglich zu einer Vermehrung des Materials führen:

Keineswegs gilt, daß je mehr man sammelt, desto größer auch das Wissen ist. Einen stetigen Wissenszugewinn gibt es nicht. [...] Ist nicht wahrscheinlicher, daß die stets weiter wachsenden Sammlungen umgekehrt anerkanntes Wissen unterminieren, bis in

\footnotetext{
5 Zedelmaier 2004, S. 192, weist im Zusammenhang der spätmittelalterlichen und frühneuzeitlichen Enzyklopädien entschieden auf die »pragmatische Funktion der Wissensaufbereitung« hin: Enzyklopädien müssen süberschaubar sein« und »schnelle Orientierung« ermöglichen.

- Friedrich 2004, S. 11

? Cahn 1991, S. 679.
} 
der Fülle des immer neu und anders Beigebrachten am Ende jede auf ein Allgemeines zielende These sich auflöst? ${ }^{8}$

Über die Ordnungsprinzipien frühneuzeitlicher Prosaerzählsammlungen wie etwa der Gartengesellschaft ist damit freilich noch wenig gesagt. Freys Sammlung orientiert sich jedenfalls nicht an den tradierten Formen der Wissensorganisation. Die in ihr kompilierten skleineren Erzählungen sind aus ihrem angestammten kommunikativen Kontext, für den man zunächst eine mündliche Gebrauchssituation annehmen wird, herausgelöst. In der Sammlung erscheint der einzelne Schwank als Teiltext unter vielen anderen Teiltexten, ${ }^{9}$ von denen er sich etwa graphisch durch hervorgehobene Kapitelüberschriften abhebt. Allerdings werden damit nur Grenzen markiert, die den einen Text vom anderen trennen. Damit ist ein Erzählfluss graphisch segmentiert, doch lässt sich aus dieser Segmentierung noch kein Hinweis auf ein Ordnungsprinzip der Sammlung ableiten. Die gesammelten Erzählungen sind nicht in einer einzig richtigen Art und Weise aufeinander zu beziehen, sondern es scheinen viele Wege zu verschiedenen Lesarten und Interpretationsmöglichkeiten auf, die offenbar nicht durch den Urheber der Sammlung hierarchisiert werden.

2.

Diesen ersten Bemerkungen zu den allgemeinen Sammlungsprinzipien kleinerer Erzählformen in der Gartengesellschaft folgt nun zunächst ein Blick auf die Paratexte von Jakob Freys Prosaerzählsammlung. In den Vorreden etwa könnte man Hinweise auf den Status des Buchs als einer Textsammlung und auf die dabei vorausgesetzten Relevanzkriterien erwarten. Frey veröffentlicht eine Sammlung von Texten, die auf dem Titelblatt als ein New hübsches und schimpflichs büchlin bezeichnet wird. Historien vnd Fabulen könnten darin gefunden werden, vil frölichs gesprechs und Schimpff reden, die Frey an vilen vnd mancherley orten, zu samen gesucht vnd colligiert (zzusammengebunden, zusammengelesen, aufgelesen, aufgesammeltı) habe. Nicht nur Kompilator und Inhalt der Sammlung werden benannt; dem Leser wird auch ein Gebrauch der gesammelten Erzählungen unter anderem inn den schönen Gärten, bey den külen Brunnen, auff den grünen Wysen und bey der Edlen Music - in Zwischenräumen und -zeiten

${ }^{8}$ Wegmann 1999, S. 264; dort bezogen auf die Sammeltätigkeit des modernen Philologen.

${ }^{9} \mathrm{Zu}$ hinterfragen ist grundsätzlich, ob ein ıSchwankı primär als Einzeltext betrachtet und analysiert werden sollte, oder ob er nicht »wie andere kleine Erzähltexte« jedenfalls literarhistorisch als »ein Pluraletantum« gelten müsste (Strohschneider 2007 [Kippfiguren], S. 167). 
also - empfohlen. ${ }^{10}$ Bereits auf dem Titelblatt ist so dem Erzählten und dem Erzählen gerade in seiner `Beiläufigkeit< ein Ort gegeben. Dennoch könnte man erwarten, dass in der Vorrede ein »Aufwertungsmotiv« zu entdecken ist, das

aus einleuchtendem Grund den Vorworten von Sammlungen [...] vorbehalten ist, [es] besteht darin, die formale - oder häufiger thematische - Einheit dessen aufzuzeigen, was a priori als künstliches oder zufälliges Sammelsurium erscheinen könnte und sich vor allem daraus ergibt, daß man [...] eine Schublade geleert hat."

In der ersten, an den Amtmann Reinbolt von Kageneck gerichteten Vorrede der Gartengesellschaft ${ }^{12}$ tritt eine solche Aufwertung unter anderem dadurch hervor, dass Frey sich selbst in die Nachfolge jener alten scribenten stellt, welche neben aller ernsthaften Beschäftigung auch sunst lustige schimpff unnd fatzwerckische bossen in schrifften und büchlin verfasst hätten. ${ }^{13}[E]$ rnsthafftige sachen beiseite legend habe er die mucken auß dem kensterlin gelassen und ein gartengespräch zusammengestellt, das den gesellschafften zü kurtzweil dienen solle. ${ }^{14}$ Nicht nur die vorgestellten Rezeptionssituationen, auch die Explikation der Produktionsbedingungen in der Vorrede akzentuiert also den sbeiläufigen`Status der in der Sammlung zusammengetragenen Erzählungen.

Ein einheitliches Sammlungsprinzip nennt die Vorrede dagegen nicht. Jakob Frey ordnet sich zwar selbst dem Typus der genannten alten scribenten zu; die damit angespielte Tradition tritt jedoch in den Texten der Sammlung selbst nicht erkennbar hervor; sie werden ohnehin größtenteils nicht als Bearbeitungen älterer Vorlagen ausgewiesen. Die einzige Quelle, die in der Vorrede genannt wird, ist Johannes Paulis Schimpf und Ernst: Daraus habe er, Frey, ungefähr zehn Texte entnommen und diese so bearbeitet, dass sie dadurch mer historischer gesehen werden. ${ }^{15}$ Der Bezug auf die bekannte und äußerst erfolgreiche Sammlung des Johannes Pauli kann auf der einen Seite sicherlich als Aufruf einer >Autorität verstanden werden; zugleich aber nimmt Frey eine signifikante Distanzierung vor und akzentuiert darin einen eigenständigen, in der Kategorie des `Historischen` gründenden Anspruch. Da nur eine einzige Quelle für einen geringen Teil der gesammelten Texte auf diese Weise explizit genannt wird, kann umso stärker ein anderer

10 Frey 1896, S. 1. Vgl. dazu Röcke 1993, S. 110-113. Röcke hebt die »üblichen Beschreibungsmuster des locus amoenus« hervor (ebd., S. 113), dessen Topoi in der Gartengesellschaft »das erste Mal [...] für eine Schwanksammlung aufgerufen werden « (ebd., S. 110). Darüber hinaus -- und das möchte ich hier zeigen - ist es vor allem die >Beiläufigkeitı des Erzählens, für das die Topoi des locus amoenus in Anspruch genommen werden.

1 Genette 2001, S. 195

12 Frey 1896, S. 3.1-5.11.

${ }^{13}$ Ebd., S. 3.7-10.

14 Ebd., S. 3.16-19.

15 Ebd., S. 4.26. 
Aspekt >historischer` Authentizität in den Vordergrund rücken: Viele der erzählten sachen nämlich seien ihm, so fährt Frey fort, selbs begegnet, viele habe er bey andern unnd allenthalben gesehen, gehört unnd erfaren. ${ }^{16}$

Schenkt man der Vorrede Glauben, dann hat Jakob Frey nur wenige der gesammelten Geschichten schriftlich aufgezeichnet vorgefunden. Tatsächlich aber besteht die Kompilation vorwiegend aus Bearbeitungen älterer Vorlagen. So beruht beispielsweise die erste Erzählung der Gartengesellschaft auf einem Text aus Heinrich Bebels Facetiae, der zweite Schwank ist die Bearbeitung eines Textes von Poggio Bracciolini, der dritte Schwank wurde wieder aus Bebels Facetiae übernommen und für die nächsten beiden Texte stand Johannes Adelphus Mulings Margarita facetiarum Pate. ${ }^{17}$ Indem Frey die literarischen Vorlagen, die sich für den größten Teil der Texte nachweisen lassen, verschweigt, übernimmt er offenbar dezidiert selbst die Verantwortung für das Erzählte. Die zweite Vorrede $[a] n$ den gütigen leser ${ }^{18}$ verstärkt diesen Eindruck noch: Jakob Frey reiht sich hier unter jene gelerte $[n]$ männer ein, ${ }^{19}$ die [v]or zeyten und noch [...] vil kurtzweiliger büchly geschrieben haben. ${ }^{20}$ Nach Paulis Schimpf und Ernst wird mit Jörg Wickrams Rollwagenbüchlein nun eine zweite Sammlung namhaft gemacht, allerdings nicht als Quelle für Texte der eigenen Sammlung, sondern allein zu deren Legitimation durch ein bekanntes Vorbild.

Zwischen die beiden Vorreden ist im ältesten erhaltenen Druck der Gartengesellschaft ein Register diser fabulen und historien der Gartengeselschafft, kurtz zu finden, ${ }^{21}$ eingeschaltet. Es handelt sich um ein mit Angabe der Folia versehenes Inhaltsverzeichnis, welches also keine inhaltliche Strukturierung des gesammelten Materials leistet und einen thematisch motivierten, willkürlichen Zugriff auf den einzelnen Text nur bedingt anhand der Auflistung der Kapitelüberschriften ermöglicht. ${ }^{22}$ Brauchbar mag es immerhin sein im Hinblick auf kolloquiale Kommunikation, wie sie in

\footnotetext{
${ }^{16}$ Frey 1896, S. $4.27 \mathrm{ff}$.

17 Vgl. Bolte 1896, S. XXV-XXIX. Frey übernimmt oder übersetzt seine Prätexte nicht lediglich, sondern erweitert und verändert sie erheblich. Vgl. Waltenberger 2006 und schon Bolte 1896, S. XXVI: "Wer jedoch nach dem eben bemerkten meinen sollte, Frey begnüge sich im wesentlichen mit der verdeutschung ausgewählter erzählungen [...], der würde seine litterarische leistung erheblich unterschätzen. Man braucht nur einen beliebigen schwank mit der lateinischen quelle zu vergleichen, um zu erkennen, dass er nicht übersetzt, sondern frei bearbeitet«. Wichtig für das Verständnis einer solchen Bearbeitungspraxis ist vor allem das Konzept des »Wiedererzählens« (Worstbrock 1999). Mit modernen Vorstellungen von literarischer Autorschaft hingegen lässt sie sich kaum angemessen beschreiben (vgl. zu vormodernen Autorschaftskonzepten bes. Müller 1993, 1995 und 1999).

18 Frey 1869 , S. 5.12-7.15.

19 Ebd., S. 5.16.

20 Ebd., S. $5.13 \mathrm{f}$.

21 Ebd., S. 153.

${ }^{22}$ Zur Tradition der facilitas inveniendi vgl. Zedelmaier 2004.
} 
den Vorreden des Buchs aufgerufen wird: Schwankerzählen kann zum Gespräch anregen und es aufrechterhalten, denn in solcherart angeregter Unterhaltung locket ye ein argument das ander herfür ${ }^{23}$ und ein Schwank den nächsten. Die Erschließung der Texte durch das Register erlaubt spontane assoziative Arrangements der Texte durch den Rezipienten: Er kann die Texte nach eigenen Interessen jeweils neu aufeinander beziehen und ist dabei kaum an eine vom Kompilator etwa intendierte Ordnung gebunden.

\section{1}

Das Buchregister erleichtert es also dem Rezipienten, Relationen zwischen den Einzeltexten herzustellen, die sich nicht aus der linearen Leserichtung ergeben. Auch die Vorreden beschränken ihn dabei kaum, denn sie enthalten, wie gesehen, kaum Hinweise auf Ordnungskriterien der Sammlung. Da aber allein aus der pragmatischen /Logik der Sammlung gerade nicht auf eine ihr inhärente >Grammatik ' geschlossen werden kann, soll nun in Lektüreexperimenten geprüft werden, auf welche Weise sich innerhalb der Sammlung übergreifende Kohärenzen bilden können. Exemplarisch möchte ich zunächst die Abfolge der ersten Texte der Gartengesellschaft betrachten. Erhält der Rezipient in ihnen bei einer sukzessiven Lektüre Anreize für übergreifende Kohärenzbildungen? Man könnte nach solchen Anreizẹn zunächst in den Kapiteltiteln suchen, wie sie ohne nennenswerte Abweichungen vom Haupttext auch im Register nacheinander angeführt sind:

1. Von eim groben närrischen bauren, der wolt junge gän $\beta$ außbrütlen

2. Von den bauren, die ein lebendigen hergott haben wolten

3. Ein jüdin hielt vil mehr vonn dem tauff dann von der beschneidung

4. Von eim schümacher, der eim bauren die füß beschneiden wolt

5. Von eim Türcken, der in der christen kirchen zů opffer gieng

$[\ldots]^{24}$

Wörtliche Rekurrenzen (bauren in Kap. 1 und 2; beschneidung und beschneiden in Kap. 3 und 4) und semantische Anklänge (hergott in Kap. 2, tauff und beschneidung in Kap. 3, christen kirchen in Kap. 5) könnten hier als Hinweise auf übergreifende Kohärenzen verstanden werden. Die Lektüre der Texte selbst zeigt jedoch, dass sich aufgrund dieser Hinweise nur sehr allgemeine und vage Gemeinsamkeiten (konventioneller Protagonistentypus des dummen Bauern; Thematisierung christlicher Rituale) konstruieren lassen. Insbesondere die Wiederaufnahme des Nomens beschneidung durch das entsprechende Verb dient nicht eigentlich als Indiz für einen Zusammenhang zwischen den Texten, sondern lenkt die Aufmerksamkeit geradezu auf ihre semantische und thematische Disparität:

${ }^{23}$ Frey 1896, S. 6.22f.

${ }^{24}$ Ebd., S. 153. 
Kap. 3:25 Ein alter Bürger rät einer schönen Jüdin zur Taufe, als Lohn stellt er ihr die Heirat mit seinem Sohn in Aussicht. Als sie fragt, worauf der christliche Glaube denn beruhe, antwortet er: auf der Taufe. Nur wer getauft sei, könne in den Himmel kommen. Ironisch gesteht die Jüdin nun den Vorrang des Christentums zu, da man den Christen in der Taufe gar nicht so viel abwaschen könne, wie den Juden bei der Beschneidung abgeschnitten werde. Ginge es nach den Frauen, so sollte den jüdischen Männern eher noch etwas angesetzt als abgeschnitten werden. Die Frauen hätten unter diesem Mangel schwer zu leiden. Der Alte merkt, dass er verspottet wird, schweigt und geht weg.

Kap. 4:26 Ein Bauer lässt sich von einem Schuhmacher ein paar neue Schuhe anfertigen. Allerdings haben diese einen Fehler: Sie sind ihm zu klein. Als der Bauer dies feststellt, wird er zornig auf den Schuhmacher und will ihm die Schuhe nicht abnehmen. Der Schuhmacher aber behauptet, der Mangel liege nicht an den Schuhen, sondern an den Füßen. Er greift sich eine Zange, packt den Bauern bei den Füßen und will sie passend beschneiden. ${ }^{27}$ Der Bauer schreit auf, bezahlt die Schuhe nun und geht nach Hause.

Die Lexemrekurrenz, noch akzentuiert durch die Wiederholungen des Wortes innerhalb der Erzählung selbst, verknüpft zwei Texte, die thematisch kaum miteinander vereinbar sind. Wer sich dennoch zur Konstruktion eines semantischen Zusammenhangs anreizen lässt, muss dies jedenfalls auf einer relativ abgehobenen Abstraktionsebene versuchen - oder er mag der Textfolge immerhin eine metasprachliche Pointe zur Ambiguität des wiederkehrenden Titelworts abgewinnen. Wenn man jedoch der Lexemrekurrenz überhaupt eine verknüpfende Funktion zurechnen will, dann ist vielleicht gar nicht in erster Linie an eine semantische Relation zu denken, sondern eventuell primär an eine /Vertextungı der von Frey in den Vorreden skizzierten Pragmatik mündlicher Unterhaltung. Das Wort beschneidung indiziert unter diesem Aspekt keinen stieferen/ Sinnzusammenhang, sondern signalisiert gerade das Aussetzen eines solchen Anspruchs. In seiner Wiederkehr bildet sich analog zur kolloquialen Kommunikationssituation die Funktion ab, assoziativ ein weiteres argument zu provozieren und auf diese Weise die >Unterhaltung ( (im doppelten Sinne) zu stabilisieren. Die schriftliche Sammlung würde damit, wenn man so will, bereits in ihren ersten Texten dezidiert die mit dem Medium des gedruckten Buchs verbundenen Ordnungs- und Einheitserwartungen enttäuschen und stattdessen für die Sinnkonstitution der in ihr kompilierten schwankhaften Kurzerzählungen auf die Bedingungen mündlicher Kommunikation zurückverweisen. ${ }^{28}$

Ähnliche Relationen zwischen aufeinander folgenden Texten lassen sich in der Sammlung sehr häufig beobachten. Immer wieder ergeben sich bei sukzessiver Lektüre assoziative Verbindungen, die sich jedoch in den meisten Fällen nicht zu einer weiter reichenden Kohärenz ausbauen lassen.

${ }^{25}$ Ebd., S. 12.14-13.5.

26 Ebd., S. 13.6-32.

${ }^{27}$ Ebd., S. 13.25; weitere Rekurrenzen in S. 13.27 (bschneidens) und 13.32 (unbeschnitten).

${ }^{28} \mathrm{Vgl}$. Bachorski 2001, S. 332f. 
Während in der mündlichen Kommunikation allerdings die sukzessiv eröffneten Freiräume assoziativer Verknüpfung durch die strenge Temporalität der Rede stets wieder verknappt werden, bietet das Medium des Buchs die Möglichkeit, die Sukzessivität zu überschreiten und textuelle Beziehungen im Vor- und Zurückblättern sowie im gezielten Nachschlagen herzustellen und abzusichern. Die lockere Assoziativität der Einzeltexte kann unter den medialen Bedingungen des Buchs als Beziehungs-Potential wahrgenommen werden, das der Rezipient - auch mithilfe des Registers - in eigenen und je neuen Arrangements aktualisieren kann. Inhaltliche oder thematische Relevanzen können in solchen retikulären Relationierungen interessenspezifisch (etwa nach Profession oder sozialem Stand der Akteure, nach bestimmten Motiven, Handlungsmustern oder Konfliktkonstellationen) gesetzt werden.

\section{2}

In einem weiteren Lektüreexperiment möchte ich eine solche Relationierung beispielhalber nachvollziehen. Ich bleibe dazu bei dem Stichwort der beschneidung, das sich mittels des Registers nicht nur in den Kapiteln 3 und 4, sondern auch in Kapitel 113 wiederfinden lässt: Von einem bauren, der unser lieben frawen geburt für die beschneidung verstund.

Kap. 113:29 Ein Bauer, der nie zur Kirche geht, wird von einem anderen Bauern gemahnt, doch wenigstens ein- oder zweimal im Jahr die Kirche zu besuchen. Gleich heute, am feyrtag unser lieben frawen geburt ${ }^{30}$ solle er damit beginnen und ein Gebet an die Mutter Gottes richten: durch dein geburt so sey mir gnedig bey deinem lieben kind! ${ }^{31}$ Der Bauer vergisst die Worte halb und schickt seine Leute zur Kirche, denn es sei unser lieben frawen tag der beschneidung und sie sollten zu Maria beten: durch dein beschneidung so sey mir genedig bey deinem lieben großvatter! ${ }^{32}$ Daraufhin weist ihn der Pfarrer zurecht: Christus selbst sei beschnitten worden, und nicht seine Mutter. Ungerührt entgegnet der Bauer, dies sei schon viel zu lange her, als dass man wissen könne, was tatsächlich geschehen sei. Ob Geburt oder Beschneidung - es liege jedenfalls weit vor seiner eigenen Lebenszeit. Und in summa, was ich nicht sihe, das glaub ich auch nit, das werden, herr pfarrher, ir mich nit überreden. ${ }^{33}$ Damit beendet er das Gespräch und lässt den Pfarrherrn stehen.

Ähnlich wie in der Erzählung von der Jüdin und dem Christen wird auch hier ein Diskurs über religiöse Rituale auf die Ebene profaner Körperlichkeit herabgezogen. Dies geschieht jedoch nicht wie dort durch eine ironische Ersetzung des rituellen Signifikats von beschneidung, sondern durch seine referenziell paradoxe Attribution. Schon diese Paradoxie wäre an sich

\footnotetext{
${ }^{29}$ Frey 1896, S. 129.23-130.23.

30 Ebd., S. $129.30 f$

3 Ebd., S. $130.1 \mathrm{f}$.

32 Ebd., S. 130.6-9.

${ }_{33}$ Ebd., S. 130.21f.
} 
hinreichend für einen komischen Effekt der Erzählung - und tatsächlich zielt Freys Prätext, eine Fazetie Heinrich Bebels, auf sie als abschließende Pointe. ${ }^{34}$ Während die Hauptfigur bei Bebel jedoch ein nobilis scholasticus ist, ${ }^{35}$ der es eigentlich besser wissen müsste und dessen Lapsus ohne weitere Konsequenzen unmittelbar als ridendi materia[] wirksam wird, ${ }^{36}$ scheint die Paradoxie bei Frey zunächst lediglich das schwankhafte Stereotyp des dummen Bauern zu bekräftigen. Die Pointe des Prätextes ist allerdings nicht mehr das Ziel des Erzählens: Sie wird auf charakteristische Weise narrativ >überspielt und selbst zum Ausgangspunkt für die Entfaltung einer tiefer greifenden diskursiven Konfliktkonstellation: ${ }^{37}$ So steht hier einerseits dem Bauern der Pfarrherr gegenüber, der über die $>$ Beschneidung Mariäく nicht wegen deren physischer Unmöglichkeit lacht, sondern sie als veritablen Glaubensirrtum verurteilt. ${ }^{38}$ Und andererseits rechtfertigt der Bauer seine Rede ausführlich - und zwar ebenfalls nicht hinsichtlich der konkret-referenziellen Paradoxie selbst, sondern indem er bestreitet, dass über historisch derart weit entfernte Sachverhalte überhaupt gültige und relevante Aussagen gemacht werden könnten. Wenn die Erzählung ihm damit auch noch das letzte Wort lässt, auf das der Pfarrherr in keiner Weise mehr zu reagieren vermag, dann steht hier offenbar mehr zur Debatte als lediglich ein witziger Lapsus: Die Schwankerzählung konfrontiert die Wahrheitsansprüche des Glaubens mit historischen und empirischen Wahrheitskriterien, die sich im 16. Jahrhundert nicht mehr ohne weiteres ersteren unterordnen lassen. Die Replik des Bauern, mit der diese Gegenposition in den Text eingespielt wird, mag zwar im Rahmen des religiösen Diskurses immer noch - als Bestätigung seiner Verstocktheit genommen werden; dass dieser Rahmen allerdings hier angelegt werden müsste, ist keineswegs mehr selbstverständlich. Weder die Kritik des Pfarrherrn noch die Argumente des Bauern treffen offenbar den Kern der Sache. Dass letzterer sich aber überhaupt rechtfertigen kann und damit jedenfalls situativ auch noch die Oberhand behält, wird durch die unverhältnismäßige Behandlung seines Irrtums durch den Pfarrherrn erst möglich: Dieser kann eine nach empirischen Kriterien leicht zu widerlegende Aussage lediglich auf der Ebene dogmatischer Lehre behandeln, deren Grundvoraussetzungen wiederum durch den Bauern in Frage gestellt werden.

Abgesehen vom gemeinsamen Stichwort der beschneidung und der daran hängenden religiösen Thematik weist das 113. Kapitel im Verhältnis zur Erzählung von der Jüdin und dem alten Bürger eine erstaunlich ähnliche

\footnotetext{
${ }^{34}$ Bebel 1931, Buch I, S.26, Kap. 58.

${ }^{35}$ Ebd., Kap. 58.1.

36 Ebd., Kap. 58.6f.

37 Vgl. ähnliche Beobachtungen zur Bearbeitung fazeter Prätexte in der Gartengesellschaft bei Waltenberger 2006 und Strohschneider 2007 (Heilswunder).

${ }^{38}$ Frey 1896, S. 130.13: Der Bauer irre sich gar weit im christenlichen glauben.
} 
Handlungs- beziehungsweise Dialogstruktur auf: ${ }^{39}$ In beiden Texten initiieren Vertreter des christlichen Glaubens durch die Mahnung zur (Re-)Integration in die Kirche einen Dialog, an dessen Ende sie jedoch verstummen müssen und eine rhetorische Niederlage erleiden. Die Rolle des Antagonisten ist dabei ganz unterschiedlich besetzt, im einen Fall durch einen kirchenfernen, eher redundant argumentierenden Bauern, im andern mit einer rhetorisch geschickten Nicht-Christin. In beiden Erzählungen aber wird ein diskursiver Konflikt exponiert, der durch die beiderseits ambivalente Figurenzeichnung und den Abbruch des Dialogs unaufgelöst bleibt. So wird im dritten Kapitel der Alte zwar als frum und als gutte[ $r]$ vatter bezeichnet, ${ }^{40}$ aber daneben auch abfällig als alt Chremes tituliert. ${ }^{41}$ Auktorial wird auf diese Weise ein Grund angedeutet, durch welchen der Rat zur Konversion - und damit zugleich das Glaubensverständnis des Christen entwertet wird: Nicht durch Glaubensargumente oder spirituelle Glücksversprechen versucht ja der Alte die Jüdin zunächst zu überzeugen, sondern durch das Versprechen einer ganz diesseitigen Belohnung, nämlich der Verheiratung mit seinem eigenen Sohn. Darin unterstellt er nicht nur seinem Gegenüber eine Prävalenz profaner Interessen vor religiösem Ethos; die knappe Charakterisierung der Jüdin als schön[] und jung[ $]^{42}$ legt den Verdacht nahe, dass sein Rat durch familiären Eigennutz motiviert sein könnte. ${ }^{43}$

Was allerdings zu Beginn der Rede des Alten nur implizit angedeutet ist, wird durch die Jüdin offen gelegt: Sie entlarvt die Koppelung des religiösen Diskurses an materielle Interessen, indem sie ironisch eine ähnliche Koppelung selbst in ihrer Antwort vollzieht. Diese responsive Struktur tritt wiederum deutlich im Vergleich zum fazeten Prätext hervor: In ihm fehlt nämlich auf der einen Seite das Motiv des Verheiratungsversprechens; auf der anderen fügt Frey der lediglich erotisch anzüglichen (und von den Umstehenden mit größtem Gelächter quittierten) Pointe der Fazetie ${ }^{44}$ Aspekte sozialen Handelns sowie einer (jedenfalls metaphorisch) ökonomisierten Sexualität hinzu und legt auf diese Weise den Rückbezug auf das Heiratsmotiv nahe: Es müssends leider unser vil, so die Jüdin, mit gedult leiden; wir sehen aber nit dester bass zum handel, das uns unsere nechste fründ und günner unsere besten und liebsten leybs narung berauben, deren wir

\footnotetext{
39 Prätext ist auch hier eine Bebel'sche Fazetie (Bebel 1931, Buch I, Kap. 2, S. 5).

40 Frey 1896, S. 13.4 und 12.20.

41 Ebd., S. 12.22. Chremes ist, insbesondere bei Terenz, der Name einer Komödienfigur: eben derjenigen eines alten geizigen Bürgers.

42 Frey 1896, S. 12.17

${ }^{43}$ Bereits in der ersten Geschichte der Gartengesellschafi (Frey 1896, S. 8.1-11.22) geht es um den vergeblichen Versuch einer reichen Witwe, ihren tölpelhaften Sohn mit einer schönen Jungfrau zu verheiraten.

44 Quoniam mallemus addi virorum nostrorum virilibus portionem quam adimi (Bebel 1931, Buch 1, Kap.2.10f., S. 5).
} 
darnach mit der zeit schwerlich manglen müssen..$^{45}$ Erst über die ironisch entlarvende Funktion wird überhaupt erklärbar, weshalb die profanisierende Rede der Jüdin, die für sich genommen ihre eigene Position desavouieren müsste und dabei lediglich das Klischee jüdischer Uneinsichtigkeit bestätigen würde, als Verspottung des Christen so wirksam sein kann, dass dieser verstummen und abziehen muss.

Innerhalb der Gartengesellschaft könnten dieser Beispielreihe vielfältige weitere schwankhafte Inszenierungen diskursiver Konflikte zwischen religiösen Geltungsansprüchen und ökonomischen Interessen hinzugefügt werden. Eine relativ große Zahl der versammelten Texte könnte auf diese Weise in einem thematischen Zusammenhang gesehen werden - allerdings ohne dass sie sich dabei in den Rahmen einer übergeordneten diskursiven Hierarchie fügen ließen und ohne dass eine einheitliche satirische oder polemische Tendenz deutlicher erkennbar würde. Am ehesten ist auf den ersten Blick eine solche Tendenz hinsichtlich des Verhältnisses zwischen Religion und Ökonomie noch dann erkennbar, wenn die Perspektivierung christlicher Riten - wie im folgenden Beispiel - über die marginale kulturelle Position der Jüdin hinaus auf die Außensicht eines Heiden verschoben wird.

Kap. 5:46 Der türkische keiser Amurates beauftragt einen Kundschafter, in Italien der christen glauben unnd ceremonien zü erkundigen. ${ }^{47}$ An Allerheiligen kommt dieser in Pisa an, besucht mit seinem Gefolge die Kirche und beobachtet zunächst aus der Distanz, wie der mönchische Priester die Messe zelebriert. Als die Gläubigen sich zum Opfergang vor dem Altar versammeln, treten auch die Türken hinzu, um die warheit über die Vorgänge dort zu erfaren. ${ }^{48}$ Nach seiner Rückkehr schildert der Kundschafter dem keiser seine Beobachtungen: In der Kirche zu Pisa habe er einen beschornen narren vor einem mit Tüchern bedeckten Steinhaufen stehen sehen. Der habe vor sich hin gemurmelt und ein langs gfecht mit ime selbs ausgetragen. ${ }^{49}$ Nach einiger Zeit habe er sich umgedreht und das Volk zusammengerufen, das eilends zu ihm gelaufen sei $^{50}$ und ihm Geld dargebracht habe. Nach der Gabe habe jeder zi lon einen lumpen küssen dürfen, ${ }^{51}$ der um den Arm des weiterhin mürrisch grummelnden Narren gewickelt gewesen sei. Auch er selbst habe ihm einen Dukaten gegeben und den lumpen geküsst, um seine narrheit ausreichend in Augenschein nehmen zu können. ${ }^{52}$ Schließlich habe der Geschorene einen Stecken aus dem Wasser gezogen und damit um sich geschlagen, so dass alle aus der Kirche geflohen seien. Christ zu werden, so resümiert der Kundschafter, könne er sich nicht leisten, denn er habe nicht genug Geld für den geschorenen Narren. Der türkische keiser korrigiert die Einschätzung seines Kundschafters: Der Geschorene sei keineswegs ein Narr, sondern vielmehr der aller witzigst gewesen. ${ }^{53}$ Ein Narr hingegen sei er selbst, der Kundschafter,

${ }^{45}$ Frey 1896, S. 12.32-13.3. Vgl. hierzu auch Röcke im vorliegenden Band, S. 290-296.

46 Frey 1896, S. 14.1-15.18.

${ }^{47}$ Ebd., S. 14.5f.

48 Ebd., S. 14.14.

49 Ebd., S. 14.23.

so Ebd., S. 14.26.

s1 Ebd., S. 14.27 und S. 14.30.

52 Ebd., S. 14.31.

${ }^{53}$ Ebd., S. 15.10. 
denn er habe sich um sein Geld betrügen lassen, ohne die Bedeutung der Vorgänge verstanden zu haben.

Im Sinne einer retikulären Relationierung durch den Rezipienten könnte diese Erzählung als strukturelle Steigerungsform einer diskursiven Konstellation aufgefasst werden, die auch im dritten und im 113. Kapitel ausgespielt wird - nun allerdings nicht diachron im Wechsel der Handlungsphasen, also durch Um- und Abbruch eines aktional-dialogischen Agon, sondern vielmehr synchron in der Überlagerung (dreier) konkurrierender Beschreibungen ein und derselben Handlung: Auf eine kurze auktoriale Situationsskizze folgt die ausführliche Schilderung dessen, was der türkische Kundschafter, der nach der religiösen Bedeutung der christlichen Riten (beziehungsweise nach der Differenz zwischen den Religionen) forscht, als närrisch-sinnloses Treiben wahrnimmt; und schließlich wird die solcherart geschilderte Narrheit als eine raffinierte und effiziente Art des Gelderwerbs durchschaut, die zwischen weißheit und Betrug schillert. Sowohl die Kapitelüberschrift (Von einem Türcken, der in der christen kirchen zu opffer gieng) ${ }^{54}$ als auch Amurates' Schlusspointe akzentuieren diese ökonomische Effizienz der religiösen Praxis: Selbst ein Heide, der mit dem Geschehen in der christlichen Kirche nichts anzufangen weiß, wird doch mit hineingezogen und verliert dort sein Geld.

In der triadischen Struktur des Textes wird die prekäre Koppelung zwischen religiöser und ökonomischer Praxis nicht, wie in einschlägigen Schwankgeschichten, als eine unmittelbare inszeniert, mithin durch den Umschlag von einer Perspektive in die konträre und vom täuschenden Schein zur Wahrheit. Die basale Differenz zwischen eigener und fremder Kultur wird nicht einfach eingesetzt, um durch sie eine solche Kippfigur noch zu schärfen, sondern um >auf der Schwelle< zwischen den gegensätzlichen diskursiven Seiten Bedingungen ihrer prekären Koppelung sichtbar machen zu können: Die extrakulturelle Position des türkischen Kundschafters erlaubt es ihm nicht unmittelbar, vorgeblich religiös sinnhaftes Handeln als listig instrumentalisiertes zu entlarven, sondern die kulturell sexterne<, gleichwohl auf die Erkundung religiösen Sinns gerichtete Beobachtung macht zunächst lediglich das Fehlen eines solchen Sinns vorstellbar: eine kollektive narrheit im Hinblick auf die Religion. Diese Beobachtung aber wird auch mit der Aufdeckung des ökonomischen Zwecks durch den türkischen keiser nicht irrelevant: Er entlarvt keine betrügerische Vortäuschung religiösen Sinns, keine schwankgemäß individuell ins Werk gesetzte Perversion des Religiösen durch das Geld, sondern ein viel subtileres Verhältnis. Aus seiner Sicht besteht die weißheit des Priestermönchs nicht eigentlich darin, dass er bestimmte religiöse Sinnvorgaben okkasionell zu seinen Gunsten manipulieren könnte, sondern darin, dass er lediglich eine kollektiv

${ }^{54}$ Ebd., S. 14. If. 
akzeptierte Handlungsrolle annimmt, die als eine närrisch-sinnlose jedenfalls >von außen ', potenziell aber auch von ihm selbst wahrgenommen werden kann: Ich sag dir, es ist ein weißheit, das sich underweilen einer zum narren machen laßt, uff das er von andern das gelt mit geschicklichkeit, got geb, sie leiden mangel oder nit, bringe. ${ }^{55}$

Was in der extrakulturellen Beobachtung zutage tritt, so könnte man folgern, ist nicht die individuelle Manipulation religiöser Praxis und religiösen Sinns zugunsten eines anderweitigen Zwecks, sondern religiöse Praxis als ein institutionalisiertes Handeln, dessen fundierende ökonomische Effizienz lediglich von der sozialen Zuschreibung als religiöses abhängt, aber nicht zugleich von der individuellen Einsicht in seinen Sinn. Selbstverständlich bleibt eine solche Lesart der Geschichte schon deshalb in der Schwebe, weil ihre Geltung davon abhängt, ob man den heidnischen Standpunkt als Voraussetzung einer robjektivierenden Außensicht akzeptiert oder den Geltungsanspruch der fremden Sicht auf das Eigene bestreitet. Andererseits lässt sich aber die heidnische Außenperspektive nicht als bloße Transposition einer konfessionellen Innenperspektive auflösen: Sie bietet wenig Anhalt für die Bestätigung eines protestantischen Standpunkts als Voraussetzung satirischer Polemik gegen katholische Riten. In einer für die Sammlung insgesamt charakteristischen Weise zielt die Geschichte auf tiefer sitzende epistemische Schichten ab und legt dabei diskursive Unabgestimmtheiten offen, die sich nicht einfach durch konfessionelle Orientierungen beheben lassen.

\section{3}

Einen Sonderfall stellt im Hinblick auf die Frage nach der Sinnkonstitution im Sammlungszusammenhang gewiss jene Gruppe von Texten dar, in denen das Dorf Beit ein Weil vorkommt: ein weder der Hölle noch dem Himmel angehörender jenseitiger Zwischenort.

Kap. 44: ${ }^{56}$ Nach der Schlacht bei Mailand und Marignano (1515) wollen die toten Landsknechte nicht mit den Schweizern auf der walstat liegen bleiben, ${ }^{57}$ sondern sammeln sich unter einer weißen Fahne mit rotem Kreuz und ziehen geordnet der Hölle zu. Die Teufel aber erschrecken vor dem Kreuzzeichen, verwehren der Truppe den Zugang zur Hölle und drängen sie dazu, den Weg zum Himmel zu nehmen. Als die guten frumen landsknecht dort Einlass begehren, ${ }^{58}$ verweigert Petrus ihnen die Aufnahme, denn da sie in ihrem Leben den Frieden gehasst hätten, verdienten sie die ewige rühe nicht. ${ }^{59}$ Daraufhin erinnert der Hauptmann der Truppe Petrus an die dreimalige Verleugnung seines Herrn: eine Treulosigkeit, derer sich die Landsknechte

\footnotetext{
${ }^{5 s}$ Ebd., S. 15.12-15.

${ }^{56}$ Ebd., S. 59.1-60.28.

57 Ebd., S. 59.5.

58 Ebd., S. 59.20.

${ }^{59}$ Ebd., S. 59.28.
} 
nie schuldig gemacht hätten. Als er droht, dies vor allen himmlischen Heerscharen zu verkünden, lenkt Petrus aus Furcht, seine Schande könnte im Himmel bekannt werden, ein und überlässt den Landsknechten ein nahe gelegenes Dorf namens Beyt ein weil, wo sie spielen, mumschantzen, zechen und frölich seyn können. ${ }^{60}$ Fortan werden alle Landsknechte nach ihrem Tod dorthin verwiesen.

Kap. 45:61 Nit lang hernach stirbt - ebenfalls in der Nähe von Mailand - ein frummer guter mann, der zwar sein Leben lang viel gegessen, aber nichts getrunken hat: Er erstickt an dem Schimmel, der von dem trockenen Brot in seinem Bauch bis in seine lufftrör und kele hinauf gewachsen ist. ${ }^{62}$ Als er an der Himmelspforte erscheint, erinnert sich Petrus noch an seine Begegnung mit den Landsknechten, will den Ankömmling deshalb ohne weiteres einlassen und stellt ihm einen Platz neben seiner bereits in den Himmel aufgenommenen Ehefrau in Aussicht. Dies behagt dem Mann jedoch ganz und gar nicht: Ist mein fraw, der böß teuffel, dainnen, so kumb oder will ich bey got nit hinein. ${ }^{63}$ Lieber wolle er nach Beit ein weil gehen und sich den Landsknechten zugesellen. Mit ihnen zecht er noch heute und begehrt nicht, in den Himmel zu kommen, denn der teufel würde in sunst mit dem bösen weib und kifechten wurm ewiglich beschissen haben. ${ }^{64}$

Kap. 109:65 Ein hinkender Schneider steht nach seinem Tod vor Petrus, der ihn nicht in den Himmel lassen will, weil er in seinem Leben den Leuten Tuch gestohlen habe. Nachdem der Schneider lang gebettelt hat, erbarmt sich Petrus schließlich doch. Zur selben Zeit will der Herrgott mit den himmlischen Heerscharen für den himmel heraus in einen garten spacieren gehn und sich erlustigen. ${ }^{66}$ Währenddessen soll der Schneider im Himmel aufpassen, dass nichts entwendet werde. Der Schneider steigt auf den vorübergehend vakanten göttlichen Thron und beobachtet von dort, wie auf Erden eine alte wüste vettel einer anderen Frau zwei Schleier stehlen will ${ }^{67}$ Da er selbst Diebstahl nun verabscheut, weil ihn dies vor Petrus in solche Bedrängnis gebracht hat, ergreift er zornig Gottes Fußschemel und schleudert ihn auf die Übeltäterin, die sofort wegläuft und die Schleier liegen lässt. Als der Herrgott ihn später seiner Anmaßung wegen zur Rede stellt und zur Strafe aus dem Himmel weist, zieht auch der Schneider nach Beit ein Weil: Da ist er noch, zecht, ist güter ding. ${ }^{68}$

Beit ein Weil ist also in diesen Geschichten ähnlich wie das Fegefeuer ein Zwischenort, der den strikten Dualismus von Himmel und Hölle, Heil und Verdammnis abmildert. Im Fegefeuer jedoch wird durch abgestufte Strafen und durch Läuterung das Urteil lediglich vorbereitet; dessen letzt- und ewiggültige Gerechtigkeit wird auf diese Weise besser nachvollziehbar. Nach Beit ein Weil hingegen gelangen diejenigen, die sich dem Dualismus aus unterschiedlichen Gründen nicht einpassen lassen. Es handelt sich nicht um ein höllennahes Purgatorium vor dem Jüngsten Gericht, sondern um ein paradiesnahes und irdisch-kurzweiliges Refugium, das den Betroffenen nach den ablehnenden Entscheidungen der zuständigen Instanzen (Teufel,

61) Ebd., S. 60.20 und S. $60.22 \mathrm{f}$.

(1) Ebd., S. 60.29-62.2.

62 Ebd., S.61.9.

6.3 Ebd., S. 61.19 f.

${ }^{64}$ Ebd., S. $62.1 \mathrm{f}$.

is Ebd., S. 124.19-126.11.

${ }^{66}$ Ebd., S. 125.5 f.

${ }^{67}$ Ebd., S. 125.14.

${ }^{6} 8$ Ebd., S. 126.10ff. 
Petrus, Gott) noch übrig bleibt. ${ }^{69}$ Seine nurmehr dem Namen nach vorläufige Existenz ${ }^{70}$ kompensiert nicht die Ambivalenzen des irdischen Lebens, sondern perpetuiert sie und hintergeht damit den unbedingten Dualismus göttlicher Gerechtigkeit. Beit ein Weil ist ein autonomer, diesem Dualismus entzogener Ort: Die Landsknechte selbst halten hier ir regiment. ${ }^{71}$

Liest man jeden der drei referierten Texte für sich, so stellt das Ausweichen auf den Zwischenort Beit ein Weil jeweils eine isolierte Pointierung ganz unterschiedlicher Herausforderungen für die Vorstellung eines Dualismus von Heil und Verdammnis dar: Bleibt auf diese Weise im einen Fall die Ambivalenz des Kriegshandwerks noch im Jenseits virulent, so wird im andern Fall die unauflösliche Bindung durch das (katholische) Ehesakrament durch seine Fortdauer noch im Himmel problematisch. Die dritte Geschichte schließlich spielt Paradoxien aus, die durch die legendenhaft konkretisierte Personalität eines allmächtigen einzigen Gottes aufgeworfen werden: Gott lässt die Menschen für eine Weile unbeaufsichtigt, um spazieren zu gehen. Der Schneider verhindert in der Zwischenzeit von Gottes Thron aus eben jene Sünde, die ihn selbst fast den Zutritt zum Himmel gekostet hätte. Gott wiederum verstößt den Schneider für die Anmaßung einer Strafgewalt, vor deren unerfüllbar universalem Anspruch er selbst längst resigniert hat, aus dem Himmel. Endgültig verdammen kann er ihn für eine Tat, die auf Erden wohl als gute zu gelten hätte, allerdings nicht. So wird der problematische Konnex von Schuld, Reue und Strafe im Horizont göttlicher Providenz durch die Einkehr des Schneiders in Beit ein Weil lediglich sistiert.

Auch wenn die Texte also als je abgeschlossene rezipiert werden können, sind zumindest die ersten beiden auf eine Weise korreliert, die innerhalb der Sammlung nur ausnahmsweise begegnet: Bereits die ersten Worte des Kapitels 45 (Nit lang hernach [...]) signalisieren einen zeitlichen Anschluss an die voranstehende Geschichte. Und wenn sich Petrus gegenüber dem um Einlass bittenden Ehemann an seine Begegnung mit den Landsknechten erinnert und durch diese Erinnerung sein gegenwärtiges Handeln motiviert wird, dann bestätigt sich darin nicht nur die Kontinuität einer Erzählwelt, sondern die zweite Geschichte zeigt sich darüber hinaus als Fortsetzung der ersten. Diese explizite und diegetische Korrelation regt die Suche nach übergreifendem Sinn an. Da die Schicksale der Landsknechte und des am Brot erstickten Mannes allerdings kaum auf einen gemeinsamen Nenner zu bringen sind, muss jedenfalls das durch den Ort Beit ein Weil dokumentierte Versagen des jenseitigen Dualismus stärker in den Vordergrund rücken.

\footnotetext{
69 Über die Aufnahme der Verstorbenen in Himmel oder Hölle wird hier - wie in vergleichbaren volkstümlichen Erzählmustern üblich - bereits unmittelbar nach deren Tod entschieden.

${ }^{70}$ Beit ist Imperativ von beiten: (er)wartens.

71 Frey 1896, S. 60.25.
} 
Signifikant ist diese Akzentuierung auch gegenüber den Prätexten in Bebels Sammlung: Bereits hier wird die zweite Geschichte zeitlich (non longo tempore post) und durch die motivierende Funktion der Erinnerung (praeteritorum memor) an die erste angeschlossen. ${ }^{72}$ Beit ein Weil existiert dort allerdings noch nicht: Petrus gewährt den Landsknechten schließlich doch Einlass in den Himmel, und der Mann, der nicht zu seiner Frau in den Himmel will, entfernt sich mit unbekanntem Ziel (nescio quorsum) ${ }^{73}$

Die weiteren beiden Texte der Gartengesellschaft, in denen das >Sonderparadies der Landsknechte genannt wird, folgen erst viele Seiten später (Kap. $61^{74}$ und 109). In ihnen wird weder explizit auf die narrative Etablierung des Ortes in Kapitel 44 zurückverwiesen, noch stehen sie untereinander und mit den ersten beiden Geschichten in einem engeren diegetischen Verhältnis. Nur ein Rezipient, der diese ersten Geschichten bereits gelesen hat, könnte alle vier Beit ein Weil-Texte retikulär korrelieren und den übergreifenden Sinnzusammenhang weiter entwickeln. Das Register unterstïtzt diese Relationierung nur sehr bedingt: Der Name Beit ein Weil kommt dort nicht vor; eine Gemeinsamkeit der Texte ließe sich allenfalls über das den Titelformulierungen implizite semantische Konzept 〉Ort im Jenseits< erkennen, ${ }^{75}$ welches dann aber noch weitere Geschichten mit einschlösse. ${ }^{76}$

4.

Die Lektüreexperimente haben zunächst den Eindruck bestätigt, dass in der Gartengesellschaft die akkumulierende und expansive 'Logik des Sammelns kaum eingeschränkt erkennbar bleibt: Der Kompilator verzichtet offenbar auf die Einführung einer durchgängigen rhetorischen oder topologischen Ordnung; stattdessen betont er den Gestus des Sammelns im Sinne Cahns. Dabei werden die Einzeltexte prinzipiell als singuläre präsentiert, und es bleibt weitgehend dem Rezipienten überlassen, sie entsprechend punktuell zu rezipieren oder auch individuell neu zu arrangieren. Diesen Ordnungsverzicht stellen die ersten Texte der Sammlung geradezu demons-

\footnotetext{
72 Bebel 1931, Buch I, Kap. 84f., S. 35f.

${ }^{73}$ Ebd., Kap. 85.7.

${ }^{74}$ Im Gegensatz zu den übrigen drei Texten bleibt in Kapitel 61 (Frey 1896, S. 75.14 76.14) die intradiegetische Existenz des Ortes unentschieden: Die abschließende auktoriale Vermutung, der listige Protagonist der Geschichte könnte nach Beit ein Weil gelangt sein, steigert hier ironisch die Naivität der vom Protagonisten überlisteten Witwe, die glaubt, er könne ohne weiteres ins Paradies reisen und von dort auch wieder zurückkehren.

${ }^{75}$ Ebd., S. $154 \mathrm{ff.:} \mathrm{der} \mathrm{landsknecht} \mathrm{wonung} \mathrm{[...],} \mathrm{wann} \mathrm{sie} \mathrm{gesterben} \mathrm{(Kap.} \mathrm{44),} \mathrm{himmel}$ (Kap. 45), Paradis (Kap.61), himmel (Kap. 109).

${ }^{76}$ Z. B. Kap. 10, 37, 39 und 129.
} 
trativ aus: Die Rekurrenz des Stichworts der Beschneidung in den Kapitelüberschriften indiziert gerade keinen semantischen Zusammenhang der Texte, sondern scheint eher die Pragmatik eines locker assoziativen mündlichen Erzählens im Buchmedium abzubilden. Der Verzicht auf eine konsequente syntagmatische Ordnung bedeutet jedoch nicht, dass die Sinnkonstitution und die Verteilung diskursiver Relevanzen gänzlich auf den Rezipienten verschoben wäre. So kann man am Beispiel der Beit ein Weil-Geschichten einerseits sehen, dass die sukzessive Abfolge der Texte durchaus fallweise semantisch funktionalisiert sein kann; und andererseits lässt sich an dieser Textgruppe besonders gut nachvollziehen, wie der Kompilator über das Sammlungssyntagma hinweg paradigmatische Akzente setzt, die die Aufmerksamkeit des Rezipienten lenken und ihn zu retikulären Relationierungen zwischen den Texten anregen können: Das Motiv des jenseitigen Zwischenortes wird von Frey nicht nur in den beiden ersten Geschichten (Kap. 44 und 45) neu gegenüber den Prätexten ergänzt, sondern auch in den beiden anderen (Kap. 61 und 109). ${ }^{77}$ Aus der Vernetzung dieser recht unterschiedlichen Geschichten ergibt sich schwerlich eine semantische Einheit; sie können aber durchaus als ein narrativer Zusammenhang rezipiert werden, der einen bestimmten Problemkomplex von verschiedenen Seiten aus in den Blick nehmen lässt, ohne dass diese Ansichten systematisiert werden müssten. Solche retikulären Relationierungen kann der Kompilator mehr oder minder deutlich anreizen und damit fallweise ein spezifisch in der `Logik (der Sammlung angelegtes diskursives Potential nutzen.

\section{Bibliographie}

\section{Quellen}

Bebel, Heinrich: Facetien. Drei Bücher. Historisch-kritische Ausgabe von Gustav Bebermeyer. Leipzig: Hiersemann 1931 (Bibliothek des literarischen Vereins in Stuttgart 276).

Frey, Jakob: Gartengesellschaft (1556). Hrsg. von Johannes Bolte. Tübingen: Laupp Jr. 1896 (Bibliothek des litterarischen Vereins in Stuttgart 209).

\section{Forschungsliteratur}

Bachorski, Hans-Jürgen: »Poggios Facetien und das Problem der Performativität des toten Witzes«, in: Zeitschrift für Germanistik N. F. 11 (2001), S. 318-335.

Bolte, Johannes: »Einleitung«, in: Jakob Frey: Gartengesellschaft (1556). Hrsg. von Johannes Bolte. Tübingen: Laupp Jr. 1896 (Bibliothek des litterarischen Vereins in Stuttgart 209), S. VII-XXXIV.

77 Bei Bebel (1931, Buch I, Kap. 19, S. 11) wird der Schneider weder gerügt noch bestraft, und er bleibt im Himmel. Der Text endet mit Gottes resignativer Klage und einem passenden Ovid-Zitat. 
Cahn, Michael: »Das Schwanken zwischen Abfall und Wert. Zur kulturellen Hermeneutik des Sammlers«, in: Merkur 45 (1991), S. 674-690.

Carruthers, Mary: The Book of Memory. A Study of Memory in Medieval Culture. Cambridge: Cambridge University Press 1990 (Cambridge Studies in Medieval Literature $10)$.

Dieckow, Peter C. M.: »Um jetzt der `Katzenborischen art Rollwagenbücher` zu gedenken - Zur Erforschung deutschsprachiger Prosaerzählsammlungen aus der zweiten Hälfte des 16. Jahrhunderts«, in: Euphorion 90 (1996), S. 76-133.

Friedrich, Udo: »Von der rhetorischen zur topologischen Ordnung. Der Wandel der Wissensordnungen im Übergang zur Frühen Neuzeit «, in: Medienheft Dossier 22 (5.10.2004), S. 9-14.

Genette, Gérard: Paratexte. Das Buch vom Beiwerk des Buches. Mit einem Vorwort von Harald Weinrich. Aus dem Französischen von Dieter Hornig. Frankfurt a. M.: Suhrkamp 2001 (Suhrkamp Taschenbuch Wissenschaft 1510).

Müller, Jan-Dirk: »Zur Einführung. Sebastian Franck: Der Schreiber als Kompilator«, in: ders. (Hrsg.): Sebastian Franck (1499-1542). Wiesbaden: Harrassowitz 1993 (Wolfenbütteler Forschungen 56), S. 13-38.

- »Auctor - Actor - Author. Einige Anmerkungen zum Verständnis vom Autor in lateinischen Schriften des frühen und hohen Mittelalters«, in: Felix P. Ingold/Werner Wunderlich (Hrsg.): Der Autor im Dialog. Beiträge zu Autorität und Autorschaft. St. Gallen: UVK 1995, S. 17-31.

- "Aufführung - Autor - Werk. Zu einigen blinden Stellen gegenwärtiger Diskussion«, in: Nigel F. Palmer/Hans-Jochen Schiewer (Hrsg.): Mittelalterliche Literatur und Kunst im Spannungsfeld von Hof und Kloster. Ergebnisse der Berliner Tagung 9.11. Oktober 1997. Tübingen: Niemeyer 1999, S. 149-161.

Röcke, Werner: »Aggression und Disziplin. Gebrauchsformen des Schwanks in deutschen Erzählsammlungen des 16. Jahrhunderts«, in: Walter Haug/Burghart Wachinger (Hrsg.): Kleinere Erzählformen des 15. und 16. Jahrhunderts. Tübingen: Niemeyer 1993 (Fortuna Vitrea 8), S. 106-129.

Schmidt-Biggemann, Wilhelm: Topica Universalis. Eine Modellgeschichte humanistischer und barocker Wissenschaft. Hamburg: Meiner 1983 (Paradeigmata 1).

Straßner, Erich: Schwank. 2., überarbeitete und ergänzte Aufl. Stuttgart: Metzler 1978 (Sammlung Metzler, Abt. E: Poetik 77).

Strohschneider, Peter: Art. »Schwank«, in: Walther Killy (Hrsg.): Literaturlexikon. Bd. 14: Begriffe, Realien, Methoden. Gütersloh/München: Bertelsmann 1993, S. 354f.

- »Kippfiguren. Erzählmuster des Schwankromans und ökonomische Kulturmuster in Strickers >Amis«, in: Jan-Dirk Müller (Hrsg.): Text und Kontext. Fallstudien und theoretische Begründungen einer kulturwissenschaftlich angeleiteten Mediävistik. München: Oldenbourg 2007 (Schriften des Historischen Kollegs, Kolloquien 64), S. $163-190$.

- »Heilswunder und fauler Zauber. Repräsentationen religiöser Praxis in frühmodernen Schwankerzählungen«, in: Beiträge zur Geschichte der deutschen Sprache und Literatur 129 (2007), S. 438-468.

Waltenberger, Michael: $»$ Einfachheit< und Partikularität. Zur textuellen und diskursiven Konstitution schwankhaften Erzählens«, in: Germanisch-Romanische Monatsschrift 56 (2006), S. 265-287.

Wegmann, Nikolaus: "Im Reich der Philologie. Vom Sammeln und Urteilen«, in: Christoph König/Eberhard Lämmert (Hrsg.): Konkurrenten in der Fakultät. Kultur, Wissen und Universität um 1900. Frankfurt a. M.: Fischer 1999 (Forum Wissenschaft: Figuren des Wissens), S. 260-272.

Worstbrock, Franz Josef: "Wiedererzählen und Übersetzen«, in: Walter Haug (Hrsg.): Mittelalter und frühe Neuzeit. Übergänge, Umbrüche und Neuansätze. Tübingen: Niemeyer 1999 (Fortuna Vitrea 16), S. 128-142. 
Zedelmaier, Helmut: ")Facilitas inveniendic. Zur Pragmatik alphabetischer Buchregister«, in: Theo Stammen/Wolfgang E. J. Weber (Hrsg.): Wissenssicherung, Wissensordnung und Wissensverarbeitung. Das europäische Modell der Enzyklopädien. Berlin: Akademie Verlag 2004 (Colloquia Augustana 18), S. 191-203.

Ziegeler, Hans-Joachim: Art. "Schwank ${ }_{2}$, in: Reallexikon der deutschen Literaturwissenschaft. Bd. 3. Berlin/New York: de Gruyter 2003, S. 407-410. 